\title{
Shear Strengthening of RC Beams by means of NSM FRP Strips: Constitutive Law of a Single Strip
}

\author{
Vincenzo Bianco (vincenzo.bianco@uniroma1.it ) \& Giorgio Monti \\ Department of Structural Engineering and Geotechnics, Sapienza University, Rome, Italy.
}

J.A.O. Barros

Department of Civil Engineering, University of Minho at Guimarães, Portugal.

\begin{abstract}
The need to provide a rational explanation to the observed peculiar failure mode affecting the behaviour, at ultimate, of a Reinforced Concrete (RC) beam strengthened in shear by Near Surface Mounted (NSM) Fibre Reinforced Polymer (FRP) strips, led the authors to develop a comprehensive numerical model for simulating the NSM shear strength contribution to RC beams throughout the entire loading process as function of the Critical Diagonal Crack (CDC) opening angle. That model was respectful of equilibrium, kinematic compatibility and constitutive laws. Despite its high level of prediction accuracy, taking into account all of the possible failure modes, as well as the interaction among adjacent strips, that model resulted relatively complex to be easily implemented in a practitioners-addressed building regulations code. Yet, it can be conveniently simplified into a more user-friendly and closed-form design formula. Crucial point of that simplification is the development of a reliable constitutive analytical law providing the single strip strength as function of the imposed end slip. This paper presents the modelling strategy adopted to determine that constitutive law, as well as its final analytical expression.
\end{abstract}

\section{INTRODUCTION}

During the loading process of a RC beam strengthened in shear by NSM FRP strips, after the occurrence of the Critical Diagonal Crack (CDC), the two parts into which the beam web divides start moving apart and the crossing strips oppose this movement by anchoring to the surrounding concrete. Due to the imposed end slip $\delta_{L i}\left(t_{n}\right)$, the force originated in each NSM strip at the section crossing the CDC is transferred to the surrounding concrete through bond (Fig. 1a). The local bond stress-slip relationship $\tau(\delta)$ simulating the mechanical phenomena occurring at the strip/adhesive interface, within the adhesive layer and at the adhesive/concrete interface, can be represented, in a simplified way (Bianco 2008, Bianco et al. 2009), by a bi-linear curve (Fig. 1b). This curve envisages that, by imposing increasing end slips to the FRP strip, cracks form instantly within the adhesive layer, orthogonally to the (inclined) tension isostatics and along the interfaces. Stresses are transferred by friction along those micro-cracks. However, by imposing increasing end slips, those cracks progressively become smoother (softening friction phase) up to the point ( $\delta_{L i}=\delta_{1}$ ) in which friction can no longer be mobilized and the strip is being pulled out without having to overcome any restraint left (free slipping phase). Even if bond is the main mechanism through which force present in the strip is transferred to the surrounding concrete, the behavior of an NSM FRP strip is more complex due to the interaction of bond with other phenomena such as concrete fracture and/or strip tensile rupture, and to the interaction among adjacent strips and between these latter and the existing steel stirrups (Bianco 2008).

\section{BOND-BASED CONSTITUTIVE LAW}

The bond behavior of an NSM FRP strip subjected to an imposed end slip $\delta_{L i}$ can be modeled (Bianco et al. 2009) by fulfilling equilibrium, kinematic compatibility, constitutive laws of the intervening materials (FRP, adhesive and concrete) and the local bond stress-slip relationship (Fig. 1b). In this way, it is possible to obtain closed-form analytical equations for both the bond-based constitutive law $V_{f i}^{b d}\left(L_{R f i} ; \delta_{L i}\right)$ of a single strip and the correspond- 
ing bond transfer length $L_{t r, f i}^{b d}\left(L_{R f i} ; \delta_{L i}\right)$ that are: the force that a strip of resisting bond length $L_{R f i}$ can transfer by bond, as function of $\delta_{L i}$ and the corresponding amount of $L_{R f i}$ along which bond is mobilized, respectively. Those analytical expressions, herein omitted for the sake of brevity (Bianco 2008), are plotted in Figure 2a-c.

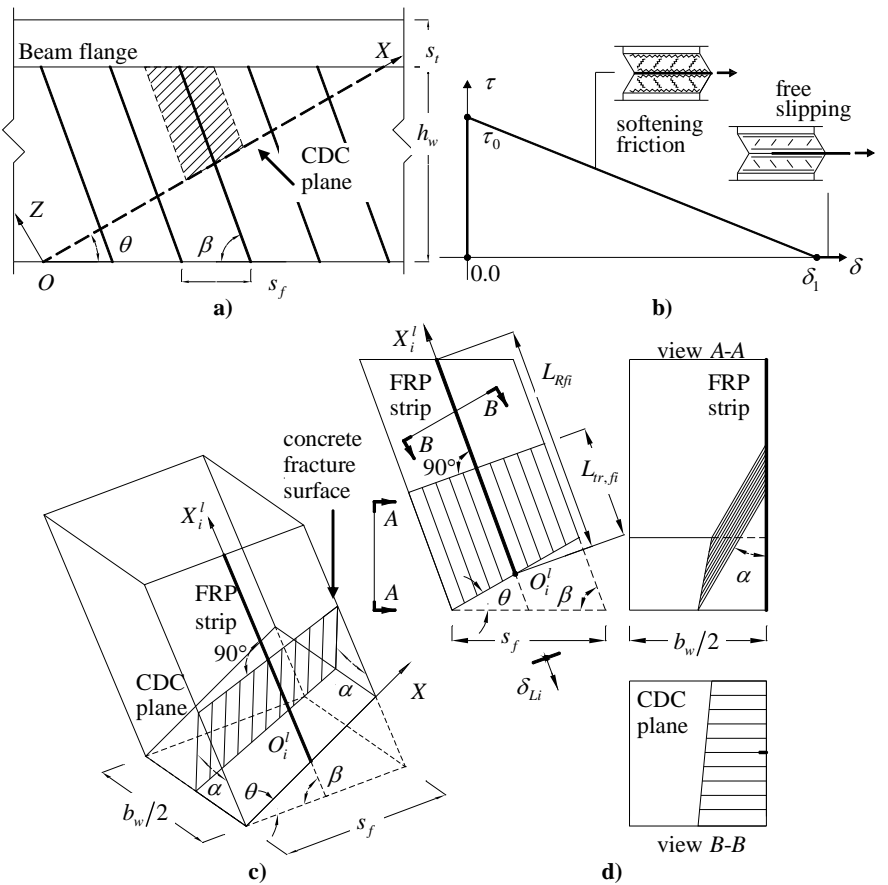

Figure 1. An NSM FRP strip: a) web strengthened side, b) local bond stress-slip relationship, c) a single strip confined to a concrete prism, d) sections of the concrete prism.

Those analytical expressions envisage, for a given $L_{R f i}$, three phases, whose limits are function of the value assumed by $L_{R f i}$ with respect to the effective bond length $L_{t r 1}$ that is the value of resisting bond length beyond which any further increase of length does not produce any further increase of the maximum force transmissible by bond. Those phases can be singled out considering the progressive migration of the invariant distribution of shear stresses and slip (Fig. 2d) from the loaded end towards the free one by increasing $\delta_{L i}$. In the first phase, the force transmitted by bond stresses increases up to reaching the peak in correspondence of $\delta_{L 1}\left(L_{R f i}\right)$ that is, for $L_{R f i}<L_{t r 1}$, the step in which the invariant distribution of bond stresses has reached the strip free end and, for $L_{R f i} \geq L_{t r 1}$, the step where $L_{t r, f i}^{b d}=L_{t r 1}$. The second phase, for $L_{R f i}<L_{t r 1}$, is characterized by a decrease of $V_{f i}^{b d}$ and a constancy of $L_{t r, f i}^{b d}$ while, for $L_{R f i} \geq L_{t r 1}, V_{f i}^{b d}$ remains constant and equal to the peak $V_{1}^{b d}$ and $L_{t r, f i}^{b d}$ goes on increasing up to $\delta_{L 2}\left(L_{R f i}\right)$. In the third phase, the invariant distribution of bond stresses progressively abandons $L_{R f i}$, whatever its value, resulting in a decrease of $V_{f i}^{b d}$ up to zero and in a constant value of $L_{t r, f i}^{b d}$ that is equal to $L_{R f i}$.
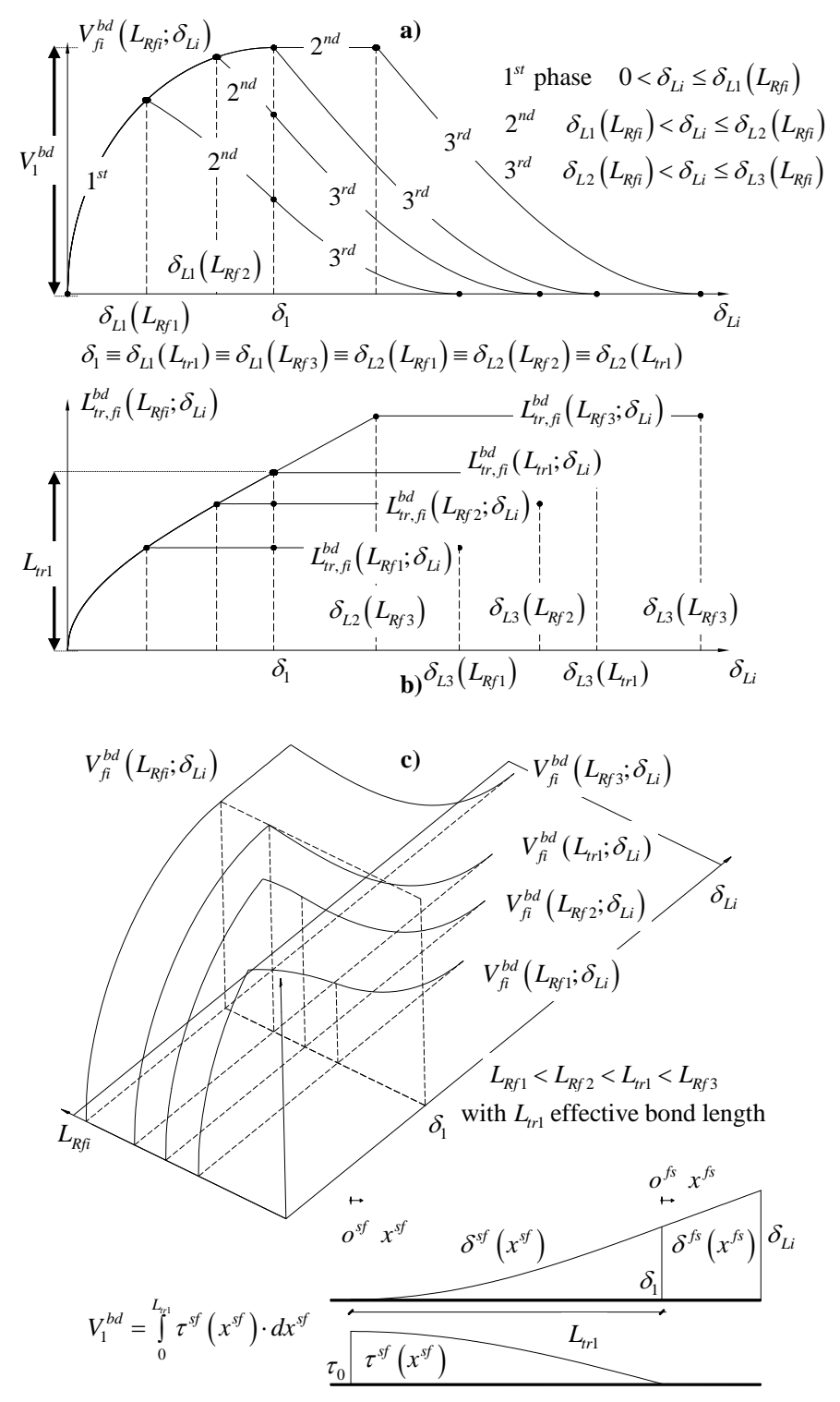

d)

Figure 2. Bond-based constitutive law of a single NSM FRP strip: a) $V_{f i}^{b d}\left(L_{R f i} ; \delta_{L i}\right)$ and b) $L_{t r, f i}^{b d}\left(L_{R f i} ; \delta_{L i}\right)$ in a bi-dimensional representation, c) $V_{f i}^{b d}\left(L_{R f} ; \delta_{L i}\right)$ in a tri-dimensional representation and d) invariant distribution of bond stress and imposed end slip.

\section{COMPREHENSIVE CONSTITUTIVE LAW}

The comprehensive constitutive law $V_{f i}\left(L_{R f i} ; \delta_{L i}\right)$ of the simple structural system constituted of an FRP strip near surface mounted on a prism of concrete is 
obtained by taking into consideration also the possibility of concrete fracture and tensile rupture of the strip itself during the loading process. In correspondence of each step $\left(t_{n}\right)$ of the loading process, the system undergoes some modifications up to reaching the corresponding equilibrium configuration $\left(q_{e}\right)$ (Fig. 3).

At the generic iteration $q_{m}$ of the $t_{n}$ load step, the transfer length is given by (Fig. 3a):

$$
\begin{aligned}
& L_{t r, f i}\left(t_{n} ; q_{m}\right)=L_{f i}^{c f}\left(t_{n-1} ; q_{e}\right)+ \\
& +L_{t r, f i}^{b d}\left[L_{R f i}\left(t_{n} ; q_{m}\right) ; \delta_{L i}\left(t_{n}\right)\right]+\Delta L_{f i}^{c f}\left(t_{n} ; q_{m}\right)
\end{aligned}
$$

that is, the sum of $L_{f i}^{c f}\left(t_{n-1} ; q_{e}\right)$ the cumulative depth of the concrete fracture as results from the equilibrium of the preceding $t_{n-1}$ load step, the $L_{t r, f i}^{b d}\left[L_{R f i}\left(t_{n} ; q_{m}\right) ; \delta_{L i}\left(t_{n}\right)\right]$ bond-based necessary transfer length corresponding to the current value of the imposed end slip and the increment $\Delta L_{f i}^{c f}\left(t_{n} ; q_{m}\right)$ of concrete fracture height at the current load step.

In correspondence of the $q_{m}$ iteration of the $t_{n}$ load step, if it happens that the concrete fracture capacity is smaller than the force transferred by bond, i.e.:

$V_{f i}^{c f}\left[L_{t r, f i}\left(t_{n} ; q_{m}\right)\right]<V_{f i}^{b d}\left[L_{R f i}\left(t_{n} ; q_{m}\right) ; \delta_{L i}\left(t_{n}\right)\right]$

the bond transfer mechanism leaps forward towards the free end of the strip and the resisting bond length reduces accordingly, up to reaching equilibrium (Fig. 3). The initial value of the resisting bond length reduces and the initial constitutive law modifies as indicated in Figure 3d for the general case in which, in correspondence of the last fracture and the contextual leap forwards, the value of the left amount of the initial resisting bond length is larger than the current value of the bond-based transfer length. In this case, in fact, concrete fracture acts just like a reducer of the initial resisting bond length and the point representative of the state of the system moves from one constitutive law to the other (Fig. 3d). On the contrary, when the concrete fracture is very deep, there can be a drastic and abrupt reduction of the resisting bond length, to which corresponds a constitutive law as that indicated in Figure 4a. In the case (Fig. 3) in which concrete fracture remains shallower and just acts like a reducer of the initial $L_{R f i}$, the resulting constitutive law is as depicted in Figure 4b. When, either following or not an initial concrete fracture, the rupture of the strip oc-

curs, the resulting constitutive law is as depicted in Figure 4c. The analytical expressions of those constitutive laws are extensively supported on the ones of the bond based constitutive law (Bianco 2008).

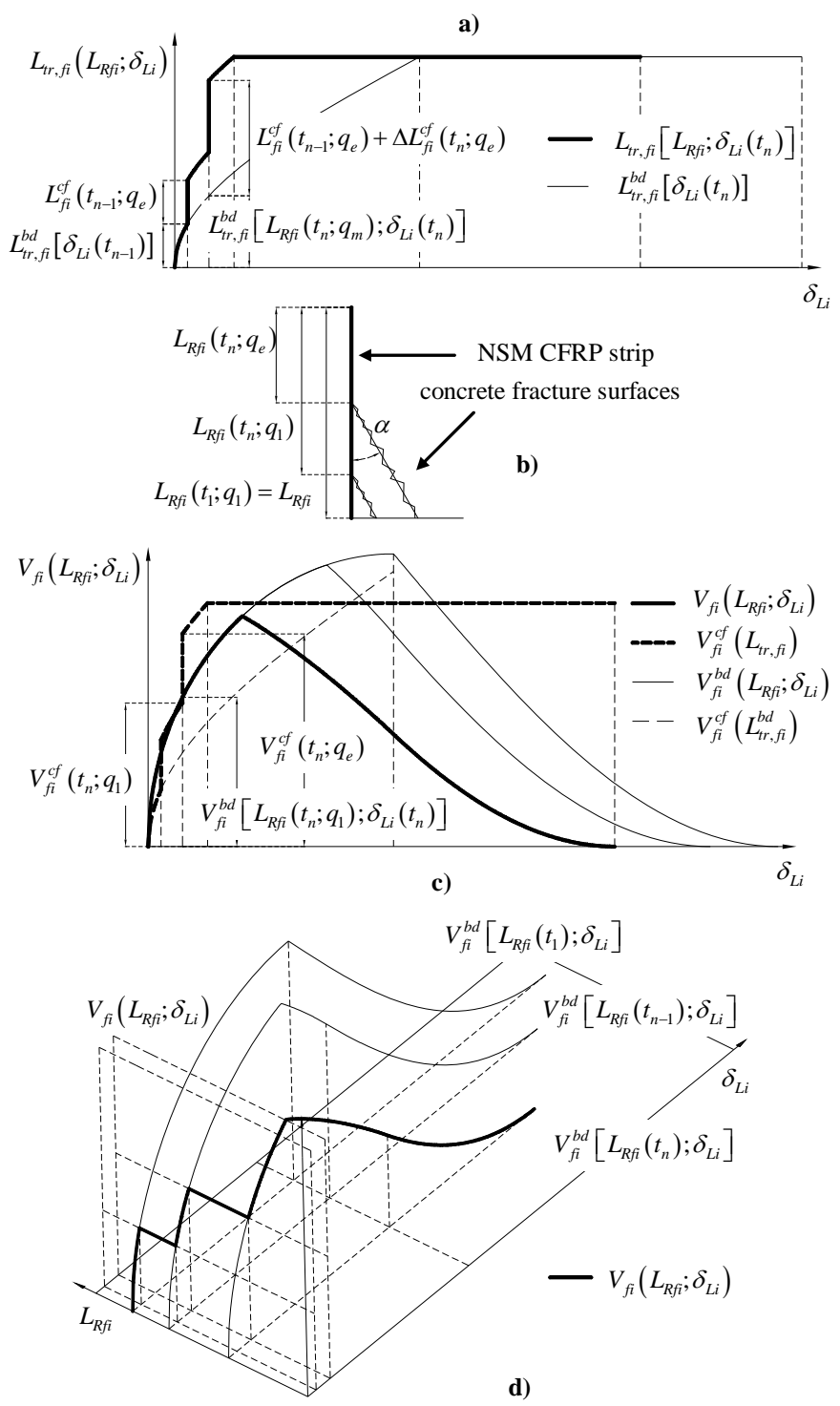

Figure 3. Comprehensive constitutive law of an NSM FRP strip: a) transfer length, b) section of the concrete prism, constitutive law in c) a 2D and d) in a 3D representation.

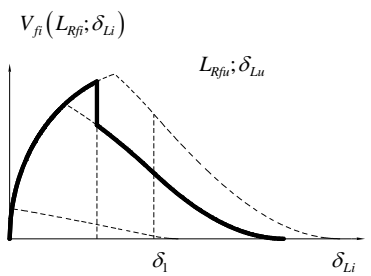

a)

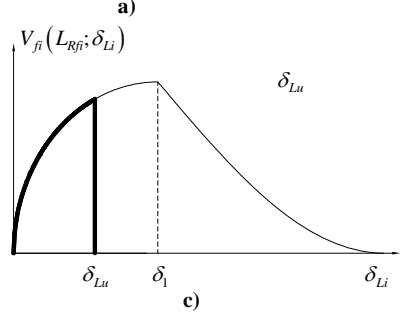

Figure 4. Comprehensive constitutive law of an NSM FRP strip confined within a prism of concrete: a) deep concrete 
fracture, b) superficial and/or absent concrete fracture, c) strip tensile rupture.

\section{CONCLUSIONS}

The correct definition of the bond-based constitutive law of an NSM FRP strip is the first step for the determination of the constitutive law of the structural system composed of an FRP strip near surface mounted on a concrete prism. Closed-form analytical equations for the bond-based constitutive law are obtained by assuming a simplified bi-linear local bond stress slip relationship and fulfilling equilibrium, kinematic compatibility and constitutive laws of the intervening materials. During the loading process of such a simple structural system as an NSM FRP strip confined to a concrete prism, in correspondence of each load step, the system undergoes some modifications that change the initial bondbased constitutive law. Nonetheless, the comprehensive constitutive law, which from an analytical standpoint does not present significant differences with respect to the bond-based one, can be determined by an iterative procedure searching for the equilibrium condition at each load step.

\section{ACKNOWLEDGEMENTS}

The authors of the present work wish to acknowledge the support provided by the "Empreiteiros Casais”, S\&P®, degussa ${ }^{\circledR}$ Portugal, and Secil (Unibetão, Braga). The study reported in this paper forms a part of the research program "SmartReinforcement - Carbon fibre laminates for the strengthening and monitoring of reinforced concrete structures" supported by ADI-IDEIA, Project $n^{0}$ 13-05-04-FDR00031. This work was also carried out under the auspices of the Italian DPC-ReLuis Project (repertory n. 540), Research Line 8, whose financial support is greatly appreciated.

\section{REFERENCES}

Bianco, V. 2008. Shear strengthening of RC beams by means of NSM FRP strips: experimental evidence and analytical modeling. PhD Thesis, Dept. of Struct. Engrg. and Geotechnics, Sapienza University of Rome, Italy.

Bianco, V., Barros, J.A.O., Monti, G. 2009. Bond model of NSM-FRP strips in the context of the shear strengthening of RC beams. ASCE Structural Journal, 135(6): 619-631. 